\title{
Extensors respond faster than flexors for the MCP joints even under the loss of the corticospinal system
}

This paper was downloaded from TechRxiv (https://www.techrxiv.org).

\section{LICENSE}

CC BY 4.0

SUBMISSION DATE / POSTED DATE

$07-12-2021 / 13-12-2021$

\section{CITATION}

Kim, Dongwon; Baghi, Raziyeh; Koh, Kyung; Zhang, Li-Qun; Hwang, Jong-Moon (2021): Extensors respond faster than flexors for the MCP joints even under the loss of the corticospinal system. TechRxiv. Preprint. https://doi.org/10.36227/techrxiv.17133251.v1

$\mathrm{DOI}$ 


\title{
Extensors respond faster than flexors for the $\mathrm{MCP}$ joints even under the loss of the corticospinal system
}

\author{
Dongwon Kim ${ }^{1,+}$, Raziyeh Baghi ${ }^{2,+}$, Kyung $\mathrm{Koh}^{2}$, Li-Qun Zhang ${ }^{2,3,4}$, and Jong-Moon Hwang ${ }^{5,6}$ \\ ${ }^{1}$ EpicWide, LLC, ${ }^{2}$ Department of Physical Therapy and Rehabilitation Science, University of Maryland, \\ Baltimore, MD, USA, ${ }^{3}$ Department of Orthopedics, University of Maryland, Baltimore, MD, USA, \\ ${ }^{4}$ Department of Bioengineering, School of Engineering, University of Maryland, College Park, MD, USA, \\ ${ }^{5}$ Department of Rehabilitation Medicine, Kyungpook National University Hospital, Daegu, Korea, \\ ${ }^{6}$ Department of Rehabilitation Medicine, School of Medicine, Kyungpook National University, Daegu, Korea \\ + : Equally contributed.
}

\begin{abstract}
Damage in the corticospinal system following stroke produces imbalance between flexors and extensors in the upper extremity including the fingers, eventually leading to flexion-favored postures. The substitution of the reticospinal tract for the damaged corticospinal tract is known to excessively activate flexors of the fingers while the fingers are voluntarily being extended. Here, we questioned whether the cortical source or/and neural pathways of the flexors and extensors of the fingers are coupled and what factor of impairment influences finger movement. In this study, a total of 7 male participants with hemiplegic stroke conducted isometric flexion and extension at the MCP joints in response to auditory tones. We measured activation and de-activation delays of the flexor and extensor of the MCP joints on the paretic side, as well as, force generation and cocontraction between the flexor and extensor. All participants generated greater torque in the direction of flexion ( $\mathrm{p}=0.017)$. Regarding co-contraction, coupled activation of the extensor is also made during flexion in the similar way to coupled activation of the flexor made during extension. As opposite to our expectation, we observed that during extension, the extensor showed marginally significantly faster activation $(\mathrm{p}=0.66)$ while it showed faster de-activation $(\mathrm{p}=0.038)$, in comparison to activation and de-activation of the flexor during flexion. But movement smoothness was not affected by those factors. Our results imply that the cortical source and neural pathway for the extensors of the MCP joints are not coupled with those for the flexors of the MCP joints and extensor weakness mainly contributes to the asymmetry between flexors and extensors.
\end{abstract}

Keywords: MCP joints, Flexion synergy, Extension synergy, Hand dexterity.

\section{INTRODUCTION}

It is common that stroke survivors have more strength and better volitional control of flexors, in comparison to extensors, at distal joints including the finger joints. In daily life, we observe that distal joints in the upper extremity of stroke survivors tend to be in the flexion posture, rather than in the neutral or extension posture. Hand opening is more difficult for stroke survivors than hand closing [1]-[3].

Though mechanical constraints including muscle shortening contributes to the asymmetry between flexors and extensors, neurological constraints are also a substantial contributor [4]. One possible neurological reason of flexion-favored postures is the flexion synergy that links shoulder abductor and flexor with the flexors of the elbow, wrist and fingers [2]. More distal joints including the wrist and finger joints are typically under the influence of the movement or posture of more proximal joints following stroke [5]. Stroke binds muscles across the upper extremity into a small number of groups and the muscles in a group tend to concurrently activate [6]-[8]. Normal hand manipulation requires lifting the arm and simply lifting the arm overcoming the gravitational force causes activation of the shoulder abductor and flexor, directly leading to flexing motion of distal joints. Indeed several researchers reported that arm support contributes to a decrease in the flexion synergy [1], [9], [10].

Another possible neurological reason of flexion-favored postures is increased activation of flexors during voluntary extension [4], [11]. Extension torque produced is cancelled out by flexion torque through excessive activation of flexors during voluntary extension. Indeed, co-activation of the flexors and extensors of the metacarpophalangeal (MCP) joints during voluntary MCP joint extension appears [4]. A 


\begin{tabular}{|c|c|c|c|c|}
\hline Subject & Sex & Age & $\begin{array}{c}\text { Paretic } \\
\text { side }\end{array}$ & $\begin{array}{c}\text { UEFM } \\
\text { score }\end{array}$ \\
\hline$S 1$ & Male & 53 & Left & 34 \\
\hline$S 2$ & Male & 54 & Right & 29 \\
\hline$S 3$ & Male & 61 & Left & 36 \\
\hline$S 4$ & Male & 40 & Left & 10 \\
\hline$S 5$ & Male & 77 & Left & 10 \\
\hline$S 6$ & Male & 47 & Right & 26 \\
\hline$S 7$ & Male & 72 & Right & 26 \\
\hline
\end{tabular}

Table 1. Demographics of participants. UEFM stands for upper-extremity Fugl-Meyer.

study reported that anesthesia of the flexors is effective in decreasing flexion torque produced during voluntary MCP extension, implying excessive flexor activation [4].

The common source of the flexion synergy and excessive activation of flexors during voluntary extension in the upper extremity might be the loss of corticospinal system input. The occurrence of a stroke damages the corticospinal tract (CST) [12]-[16]. Alternative pathways to the damaged CST includes the ipsilateral corticospinal tract [17], vestibulospinal tract [18] and reticulospinal tract [16]. In particular, use of the reticulospinal tract facilitates flexors while suppressing extensors [19], [20]. This pathway excites the resting potential of the motoneurons closer to their thresholds via interneuronal excitation [21]. Then, a question arises here: are flexors and extensors activated and de-activated in a synchronized manner in the presence of the loss of corticospinal system input? Or are flexors and extensors controlled separately while extensor weakness is the main reason of asymmetry between flexors and extensors [4], [22].

In this study, we had participants with hemiplegic stroke respond to auditory tones by flexing and extending the MCP joints in the isometric condition. We measured response time of the MCP joints on the paretic side as well as force generation and corresponding muscle activity. A previous study investigated response time of the paretic wrist joint and corresponding muscle activity during flexion and extension [23]. But wrist and finger movements are made through different cortical activation [24], leading to different recovery time [unpublished]. It would be worth revisiting neural antagonism between flexors and extensors by investigating MCP joint movement.

\section{Participants}

A total of 7 hemiplegic male volunteers post stroke (age: $61.57 \pm 12.03$ (S.D) years; impaired side (L/R): 4/3, time since stroke: $7.63 \pm 6.51$ (S.D) month) participated in the study. The inclusion criteria were moderate-to-severe upper extremity impairment (upper extremity FuglMeyer (UEFM) score $<50$ ) and sufficient cognitive/language abilities to follow instructions during the experiment (Mini-Mental Status Score >22). We excluded volunteers who had severe shoulder pain, relevant musculoskeletal injury, or fixed contraction deformity in the upper extremity. None of the participants received pharmacological medications for spasticity and tone (i.e. Botulinum toxin injection to the upper limb) in the 5 months before the experiment. Each participant gave written informed consent approved by the Institutional Review Board of the University of Maryland, Baltimore. Table 1 presents the demographics of participants.

\section{Procedure}

Participants sat on a height-adjustable chair with a back support and their paretic hand was placed on a rotatable rigid plate that was connected to an electrical motor with an encoder (Maxon Brushless EC60, Sachseln, Switzerland). A torque transducer (Transducer Techniques TRT-100, Temecula, USA) was placed between the plate and motor. The metacarpophalangeal (MCP) joints of the digits II-V were aligned with the rotation axis of the plate. The hand and forearm were supported being tightly fixed to the device using rigid mechanical blocks with cushion and Velcro straps. The arm posture was maintained with shoulder adduction of $45^{\circ}$, shoulder flexion of $45^{\circ}$, elbow flexion of $90^{\circ}$, and wrist flexion of $0^{\circ}$, respectively. Wireless EMG electrodes (Delsys, Boston, USA) were placed on the flexor digitorum superficialis (FDS) and extensor digitorum superficialis (EDS) muscles.

The experiment consisted of two sessions. The first session was to evaluate the ability of participants to flex and extend the MCP joints voluntarily. Participants were instructed to move their MCP joints back and forth as much as possible. The hand plate was backdriveable while it measured the angle of the MCP joints of the digits II-V. The second session was to determine timing

\section{METHODS}


and muscle activity during MCP joint flexion and extension. The MCP joints of the digits II-V were passively locked by the motorized resistance when the joints were at the neutral position. Participants were first requested to flex and relax the MCP joints against motorized resistance in response to auditory tones. Three pairs of tones were given. Participants were asked to flex maximally, as quickly as possible, in response to the first tone of each pair, and relax as quickly as possible after the second tone. These pairs were placed 20 seconds apart to alleviate fatigue, and the first and second tones of the three pairs were gapped by 3,2 and 4 seconds, respectively, to reduce the learning effect on timing. Verbal cues were presented prior to those three trials to get the participant ready to respond. Next participants were requested to extend and relax the MCP joints against motorized resistance in response to auditory tones. Three pairs of tones were given as well. A practice period was given to each participant to get familiar to the instruction.

Data acquisition of joint angle and EMG signals were conducted in a LabVIEW environment. The sampling rate was set at $1000 \mathrm{~Hz}$.

\section{Analysis}

The delays were determined by the time gap between the occurrences of the auditory tones and initiation/termination. Delays in initiation and termination of FDS and EDS were evaluated using EMG responses versus a predefined threshold (3 standard deviations above the mean of EMG during the rest period) [25], as seen in Fig. 1. EMG signals were low-pass filtered at $225 \mathrm{~Hz}$, rectified and low-pass filtered at $10 \mathrm{~Hz}$ using MATLAB (MathWorks, Natick, MA) to produce linear envelopes (LEs) [25]. EMG LEs were subtracted by the mean of the EMG LE during the rest period.

To quantify the degree of the activation of the antagonist muscle while the agonist muscle is voluntarily activated, antagonist activation ratios were calculated, respectively, as a ratio of the degree of the activation of FDS or EDS when they are the antagonist muscle to that of FDS or EDS when they are the agonist muscle. The mean of the EMG LE of the agonist muscle (EDS or FDS) was obtained between the initiation and termination of the agonist muscle for each trial, while the mean of the EMG LE of the antagonist muscle was obtained during the same time period at each trial, during flexion and extension, respectively. The antagonist activation ratios were computed for each trial by dividing the mean of the EMG LE of the antagonist

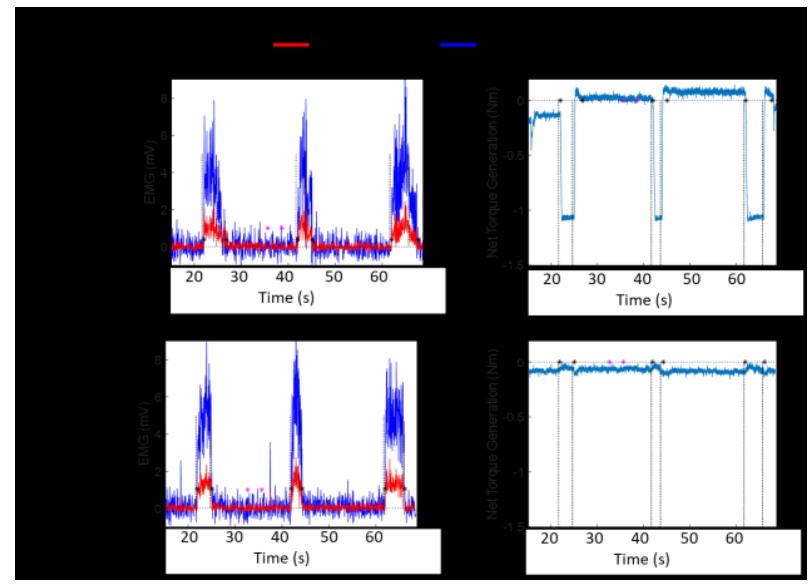

Fig 1. EMG and torque responses of a representative subject during voluntary flexion and extension, respectively. Black dashed lines indicate the times points of auditory tones occurring. The black asterisks indicate the time points that are registered by responses of the subjects versus a predefined threshold. The predefined threshold (3 standard deviations above the mean of EMG during the rest period) is based on the rest period defined by the pink asterisks.

muscle for a trial by the mean of the EMG LE of the antagonist muscle when it was the agonist muscle for the corresponding trial.

Torque generation was also considered by averaging raw torque values from the torque transducer between the time points when the response of the agonist muscle (EDS or FDS) goes beyond and down the predefined threshold, during voluntary flexion or extension for each trial, respectively.

The spectral arc length was employed to evaluate the smoothness of the movement of the MCP joints [26], [27]. Computation of the spectral arc length is carried out based on the angular velocity profile of the MCP joints $\omega_{M C P}(t)$ as

$$
\begin{aligned}
& \eta_{s a l} \triangleq-\int_{0}^{\omega_{c}} \sqrt{\left(\frac{1}{\omega_{c}}\right)^{2}+\left(\frac{d \widehat{W}_{M C P}(\omega)}{d \omega}\right)^{2}} d \omega, \\
& \widehat{W}_{M C P}(\omega) \triangleq \frac{\widehat{W}_{M C P}(\omega)}{\widehat{W}_{M C P}(0)},
\end{aligned}
$$

where $\widehat{W}_{M C P}(\omega)$ is the Fourier magnitude spectrum of $\omega_{M C P}(t)$ and $\left[0, \omega_{c}\right]$ is the frequency band where active movement is considered to occur.

In this study, $\omega_{c}$ was set to be $4 \pi \mathrm{rad} / \mathrm{s}$, or $2 \mathrm{~Hz}$, which covered active movements of the participants in this 
study. The angular velocity of the MCP joints was obtained through numerical differentiation of the angle of the MCP joints and zero-phase filtering with a 4thorder, 2-Hz Butterworth low-pass filter.

A repeated-measures analysis of variance (RM ANOVA), with trial (3 trials) and motion (flexion and extension) as the independent variables, was used to evaluate performance changes across repeated measurements. If the sphericity assumption in ANOVAs was violated, then Greenhouse-Geisser adjusted p-values were used. All analyses were preceded by Shapiro-Wilk tests of normality and their results were employed only when normality was not violated. RM ANOVA was applied to delays in initiation and termination of the agonist and antagonist muscles, respectively, antagonist activation ratio, and torque generation.

For delays in initiation and termination of the antagonist muscle, we excluded the cases that the response of the antagonist muscle looked like noise (i.e. the activation level did not remain above the threshold for more than 0.1 seconds) and that activation of the antagonist muscle was made after de-activation of the agonist muscle from analysis.

Correlation analyses of movement smoothness with delays in initiation and termination, antagonist activation ratio, and torque generation were performed to investigate whether those factors considered in this study influenced movement smoothness.

The statistical analyses were performed with SPSS version 20.0 (SPSS Inc., Chicago, USA). The significance level was set at 0.05 .

\section{RESULTS}

Repeated-measures ANOVAs were conducted on each measure with trial (3 levels: Trials 1-3) and motion (2 levels: flexion and extension) as within-subjects variables.

ANOVA on delay of initiation of the agonist muscle showed a marginally significant main effect of motion $\left[\mathrm{F}(1,6)=5.016 ; \mathrm{p}=0.066 ; \eta_{p}^{2}=0.455\right]$. We found no significant main effect of trial ( $p>0.1)$ and no significant interaction effect $(p>0.1)$. These results imply that activation of EDS is marginally faster than FDS in response to auditory cues and that there is no learning

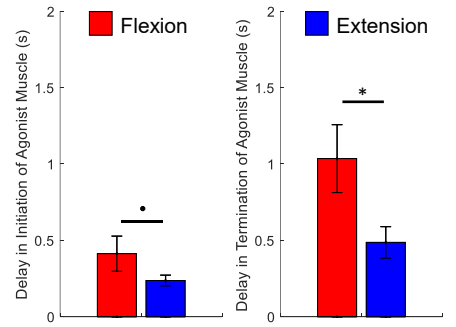

(a)

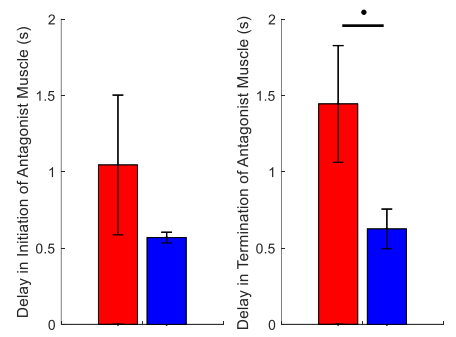

(b)

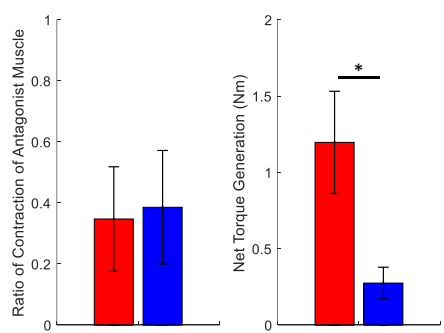

(c)

Fig 2. (a) The delays of initiation and termination of the agonist muscle, (b) the delays of initiation and termination of the antagonist muscle, and (c) the ratios of antagonist muscle activation and the net torque, during voluntary flexion and extension. Asterisks indicate statistical significance $(\mathrm{p}<0.05)$ and marginally statistical significance $(p<0.1)$. Error bars are \pm 1 standard error of the mean.

effect as trials advanced. ANOVA on delay of termination of the agonist muscle showed a significant main effect of motion $[\mathrm{F}(1,6)=7.070 ; \mathrm{p}=0.038$; $\left.\eta_{p}^{2}=0.541\right]$. No significant main effect of trial $(\mathrm{p}>0.1)$ and significant interaction effect $(p>0.1)$ were reported. These results imply that de-activation of EDS is faster than FDS and that there is no learning effect.

For delays in initiation and termination of the antagonist muscle, we excluded 4 participants from analysis for the flexion trials, while we excluded 2 participants for initiation delay and 3 participants for termination delay from analysis for the extension trials (refer to Fig. 3). De-activation of the antagonist muscle in one subject (S3) ended before the cues of termination. ANOVA on 

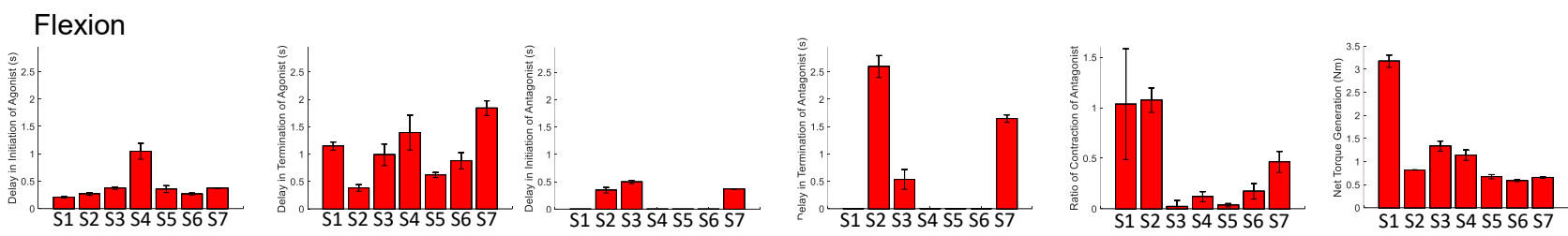

\section{Extension}
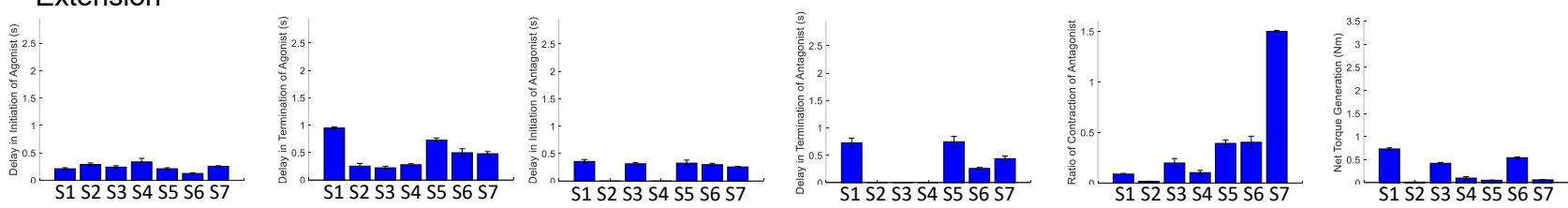

Fig 3. Individual results: the delays of initiation and termination of the agonist muscle, delays of initiation and termination of the antagonist muscle, and ratios of antagonist muscle activation and the net torque, during voluntary flexion and extension. Error bars are \pm 1 standard error of the mean.

delays in initiation and termination of the antagonist muscle found only one marginally significant main effect of motion in delay in termination $[\mathrm{F}(1,1)=45.451$; $\left.\mathrm{p}=0.094 ; \eta_{p}^{2}=0.978\right]$, suggesting that de-activation tends to end fast in EDS than FDS.

ANOVA on antagonist muscle activation ratio revealed no significant effects of trial and motion $(p>0.1)$. This indicates that the activation level of FDS when it is the antagonist muscle versus when it is the agonist muscle is not significantly different than the activation level of EDS when it is the antagonist muscle versus when it is the agonist muscle.
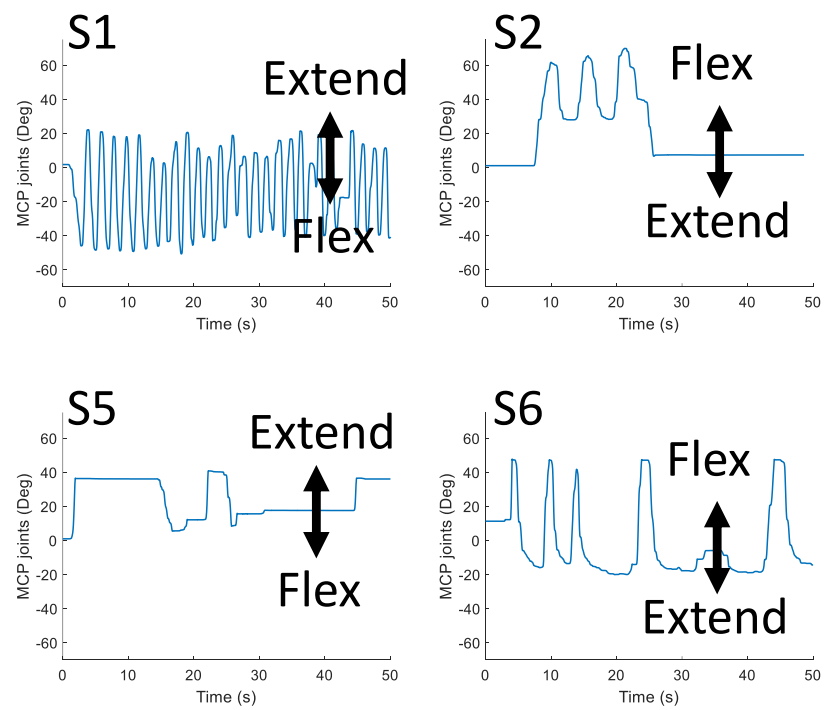

ANOVA on net torque generation showed a significant main effect of motion $[\mathrm{F}(1,6)=10.770 ; \mathrm{p}=0.017$; $\left.\eta_{p}^{2}=0.642\right]$. No significant main effect of trial $(\mathrm{p}>0.1)$ and significant interaction effect $(\mathrm{p}>0.1)$ were reported. These results imply that greater net torque was produced during flexion than during extension and that there is no learning effect.

Fig. 4 exhibits movements of the MCP joints of individual participants and the trend of movement smoothness versus the total UEFM score. Correlation analysis showed that movement smoothness was correlated with the total UMFM score $(p=0.029$, $\mathrm{r}=0.804)$. We found that movement smoothness was not
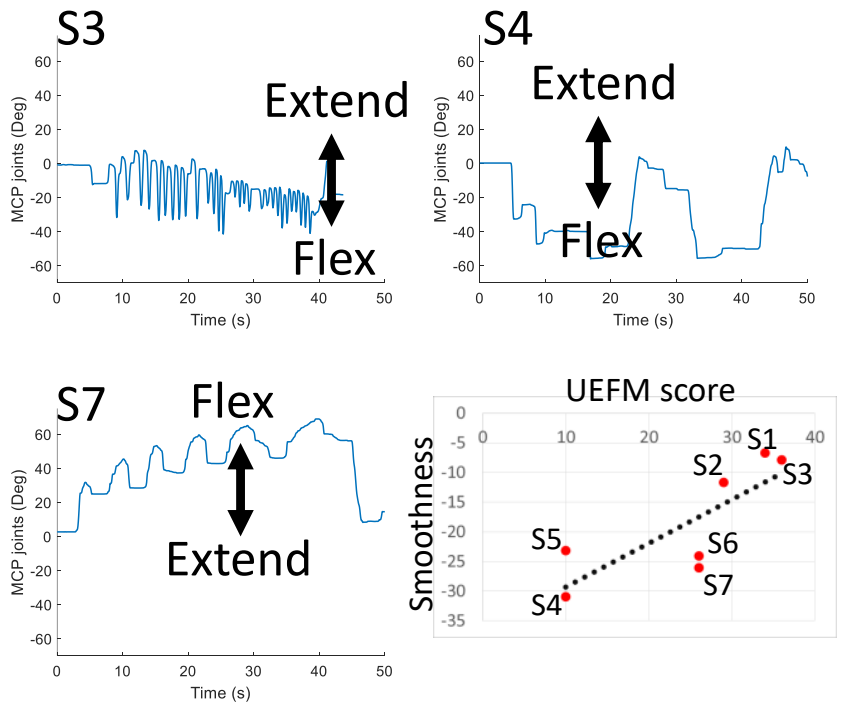

Fig 4. Free movements of the MCP joints of individual participants. The last plot presents the trend of movement smoothness versus the total UEFM score. 


\begin{tabular}{|c|c|c|c|c|c|c|c|c|c|}
\hline & UEFM & $\begin{array}{c}\text { Initiation of } \\
\text { FDS } \\
\text { (Flexion) }\end{array}$ & $\begin{array}{c}\text { Termination } \\
\text { of FDS } \\
\text { (Flexion) }\end{array}$ & $\begin{array}{l}\text { Antagonist } \\
\text { ratio } \\
\text { (Flexion) }\end{array}$ & $\begin{array}{l}\text { Net torque } \\
\text { (Flexion) }\end{array}$ & $\begin{array}{c}\text { Initiation of } \\
\text { EDS } \\
\text { (Extension) }\end{array}$ & $\begin{array}{c}\text { Termination } \\
\text { of EDS } \\
\text { (Extension) }\end{array}$ & $\begin{array}{c}\text { Antagonist } \\
\text { ratio } \\
\text { (Extension) }\end{array}$ & $\begin{array}{l}\text { Net torque } \\
\text { (Extension) }\end{array}$ \\
\hline Smoothness & $\begin{array}{l}\mathrm{r}=0.804 \\
\mathrm{p}=0.029\end{array}$ & $\begin{array}{c}\mathrm{r}=-0.398 \\
\mathrm{p}=0.446\end{array}$ & $\begin{array}{c}\mathrm{r}=-0.321 \\
\mathrm{p}=0.503\end{array}$ & $\begin{array}{l}\mathrm{r}=0.013 \\
\mathrm{p}=0.980\end{array}$ & $\begin{array}{l}\mathrm{r}=0.587 \\
\mathrm{p}=0.301\end{array}$ & $\begin{array}{l}\mathrm{r}=0.152 \\
\mathrm{p}=0.885\end{array}$ & $\begin{array}{l}\mathrm{r}=0.003 \\
\mathrm{p}=0.997\end{array}$ & $\begin{array}{c}\mathrm{r}=--0.273 \\
\mathrm{p}=0.711\end{array}$ & $\begin{array}{c}\mathrm{r}=-0.519 \\
\mathrm{p}=0.596\end{array}$ \\
\hline
\end{tabular}

Table 2. Results (Pearson's correlation coefficient and p-value) of correlation analysis of the smoothness of MCP joint movement with the total UEFM scores, delays in initiation and termination, antagonist muscle ratio,

significantly correlated with any other factors we investigated in this study $(\mathrm{p}>0.1)$. The results would mean that delays of initiation and termination of flexors and extensors, ratio of antagonist muscle activation, and the net torque generation do not affect movement smoothness.

\section{DISCUSSION}

Originally, corticospinal motor inputs in people without neurological damage are nearly equally distributed between flexors and extensors [28]. But stroke takes the ability to maintain their posture at neutral away from the victims. While victims with the parietal lobe lesioned are likely to hold their fingers extended and have difficulty in flexing their fingers [29], victims with a damaged corticospinal system are likely to have their fingers flexed and show difficulty in extending their fingers [30]. In this study, we investigated activation/deactivation timing of the flexor and extensor of the MCP joints on the paretic side as well as force generation in an effort to identify reasons of asymmetric motions. Though reasons of asymmetry in extensors and flexors could be found in spasticity [4] and altered motor coordination [31], [32], we focused on changes in neurological mechanisms of flexors and extensors following stroke.

Above all, we observed that participants generated a greater amplitude of torque at the MCP joints during flexion than during extension $(\mathrm{p}<0.05)$, as seen in Fig. 2 and Fig. 3. These results would mean that all participants might have a deficit in generating corticospinal system inputs [30], [33]. Those participants may employ the reticulospinal tract, as a substitute of the corticospinal tract. Use of the reticulospinal tract results in increased flexor activity, by increasing the resting potential of the motoneurons up to the threshold [21]. The reticulospinal tract is even know to suppress extensor activity [19], [20]. This might be why the net torque is generated greater during flexion in comparison to during extension.
We would have hypothesized that the degree of activation of the antagonist muscle or FDS increases during extension, while that of the antagonist muscle or EDS decreases durng flexion due to the influence of the use of the reticulospinal tract. But this is not the case for our results. We observed that the activation level of FDS during extension versus that during flexion is not significantly different than the activation level of EDS during flexion versus that during extension $(p>0.1)$, as presented in Fig. 3. A previous study suggested that persistent and inappropriate flexor activation plays a role in limiting voluntary MCP joint extension [10]. However the study did not present a comparison with extensor activation during voluntary MCP joint flexsion; it would not be reasonable to assert that difficulty in extension merely originated from eccessive activation of the flexor. This study revealed that the extesor of the MCP joints is also activation during flexion. However, we should note that the strength of force generated by the flexors of the MCP joints is different from those by the extesors of the MCP joints. Asymmetry in torque generation could be attributed to the weakness of the extensor muscles [4], [22]. The magnitude of torque that the MCP joint extensors generate is typocally too small to overcome that by the $\mathrm{MCP}$ joint flexors during extension.

The highlighted finding of this study is perhaps that the termination of EDS during extension is significanly faster than that of FDS during flexion $(\mathrm{p}<0.05)$. Also we found that 5 of the 7 participants showed faster activation of EDS than FDS, in response of auditory cues (see Fig. 3). Even the remaining participants (S1 and S2) did show a small difference between intiniation delays of the flexor and extensor. We would have hypothesized that the activation and de-activation of the flexor are faster at least in comparison to the extensor, since the prevailing tract, the reticulospinal tract, favors and facilitates flexors. Significant differences between intiniation and termination delays of the flexor and extensor would indicate the existence of separate cortical sources and neural pathways for the extensors of the MCP joints. As mentioned above, our results are relatively opposite to the theory that the use of the reticulospinal tract results in eccessive activation of the 
flexor during voluntary extension. The theory natually leads to an assumption that the cortical source and neural pathway for the extensors of the MCP joints are strongly coupled with those for the flexors of the MCP joints, in particular, timewise. But significant differences in intiniation and termination delays nullify the assumption.

Differences in intiniation and termination delays might be due presumably to different neural routes to the motor neuron pools from the brain. The first possibility of different neural routes resulting in differences in intiniation and termination delays can be found in the assumption that MCP flexors are dominantly governed by the reticulospinal tract, while MCP extensors are dominantly governed by the corticospinal tract. If this assumption works, there is surely a clear difference in pathways for extensors and flexors. To activate MCP flexors, motor command goes down to the motor neuron pool through the corticoreticular tract and reticulospinal tract. Meanwhile, to activate MCP extensors, motor command goes down to the motor neuron pool through the corticospinal tract. Neurons in the corticospinal tract synapse on neurons in the spinal cord, contacting with skeletal muscle to cause the muscle to contract. In terms of conduction velocity, that of the corticospinal tract ranges between 66 and $72 \mathrm{~m} / \mathrm{s}$ [34], whereas that of the reticulospinal tract ranges between 16 and $80 \mathrm{~m} / \mathrm{s}$ [35]. It might be unreasonable to merely attribute differences in intiniation and termination delays to the difference in conduction velocity, but motoric outputs could the reason of differences in intiniation and termination delays from uses of the corticospinal tract and reticulospnal tract. A study showed the flexor of the paretic MCP joints in stroke survivors that would be assumed to be primarily under control of the reticulospinal tract is activated and de-activated slowly than that of the non-paretic MCP joints that would be assumed to be under control of the corticospinal tract [25].

The second possibility of different neural routes resulting in differences in intiniation and termination delays could be that MCP flexors and extensors have different pathways to the motor neuron pools from either of the reticulospinal tract or the corticospinal tract. It is accompanied by the assumption that the reticulospinal tract and corticospinal tract compete [36], meaning that differences in intiniation and termination delays might not occur in the tract level. It is known that the median nerve innervates MCP flexors while the radial nerve innervates MCP extensors [4], [37].
In this study, we found that movement smoothness was not influenced by activation/de-activation delays and activation ratio of the antagonist muscle which we have focused on (refer to Table 2). These results implicily indicate that the weakness of extensors for the MCP joints is the main reason of asymmetry between flexors and extensors. It has been demonstrated that muscle weakness is the main contributor to difficulty in extending joints [4], [22]. Extensor weakness leads to the lack of torque enough to overcome torque produced by flexors in addition to. strong spasticity and increased stiffness of flexors of the MCP joints [4] [38].

Limitations of this study include the experimental design. Flexion trials always preceeded extension trials. We would have assumed that a learning effect might be influncial on experimental results. However, RM ANOVA found no main effects of trial across the measures evaluated in this study. This means that the learning effect did not affect the results of the experiment in the study.

\section{Sources of Funding}

This research study was supported by the National Institute on Disability, Independent Living, and Rehabilitation Research (Grant \# 90DP0099). The first author was also supported by the University of Maryland Baltimore Institute for Clinical and Translational Research (ICTR) which is funded in part by Grant Number TL1 TR003100 from the National Center for Advancing Translational Sciences (NCATS) a component of the National Institutes of Health (NIH), and NIH Roadmap for Medical Research.

\section{Disclosures}

L-Q Zhang has an ownership in Rehabtek LLC, which received U.S. federal funding in developing the rehabilitation robot used in this study.

\section{REFERENCES}

[1] M. D. Ellis, Y. Lan, J. Yao, and J. P. A. Dewald, "Robotic quantification of upper extremity loss of independent joint control or flexion synergy in individuals with hemiparetic stroke: a review of paradigms addressing the effects of shoulder abduction loading," $J$. Neuroeng. Rehabil., vol. 13, no. 1, pp. 1-11, 
2016, doi: 10.1186/s12984-016-0203-0.

[2] T. E. Twitchell, "The restoration of motor function following hemiplegia in man," Brain, vol. 74, no. 4, pp. 443-480, 1951, doi: 10.1093/brain/74.4.443.

[3] D. G. Kamper and W. Z. Rymer, "Impairment of voluntary control of finger motion following stroke: Role of inappropriate muscle coactivation," Muscle and Nerve, vol. 24, no. 5. pp. 673-681, 2001, doi: 10.1002/mus.1054.

[4] D. G. Kamper, R. L. Harvey, S. Suresh, and W. Z. Rymer, "Relative contributions of neural mechanisms versus muscle mechanics in promoting finger extension deficits following stroke," Muscle and Nerve, vol. 28, no. 3, pp. 309-318, 2003, doi: 10.1002/mus. 10443.

[5] L. M. McPherson and J. P. A. Dewald, "Differences between flexion and extension synergy-driven coupling at the elbow, wrist, and fingers of individuals with chronic hemiparetic stroke," Clin. Neurophysiol., vol. 130, no. 4, pp. 454-468, 2019, doi: 10.1016/j.clinph.2019.01.010.

[6] R. F. Beer, J. P. A. Dewald, and W. Z. Rymer, "Deficits in the coordination of multijoint arm movements in patients with hemiparesis: evidence for disturbed control of limb dynamics," Exp. Brain Res., vol. 131, no. 3, pp. 305-319, 2000, doi: 10.1007/s002219900275.

[7] J. Roh, W. Z. Rymer, E. J. Perreault, S. B. Yoo, and R. F. Beer, "Alterations in upper limb muscle synergy structure in chronic stroke survivors," J. Neurophysiol., vol. 109, no. 3, pp. 768-781, 2013, doi: 10.1152/jn.00670.2012.

[8] Z. A. Wright, W. Z. Rymer, and M. W. Slutzky, "Reducing abnormal muscle coactivation after stroke using a myoelectric-computer interface: A pilot study," Neurorehabil. Neural Repair, 2014, doi: 10.1177/1545968313517751.

[9] R. F. Beer, J. P. A. Dewald, M. L. Dawson, and W. Z. Rymer, "Target-dependent differences between free and constrained arm movements in chronic hemiparesis," Exp. Brain Res., vol. 156, no. 4, pp. 458-470, 2004, doi: 10.1007/s00221-003-1807-8.

[10] B. T. Iwamuro, E. G. Cruz, L. L. Connelly, H. C. Fischer, and D. G. Kamper, "Effect of a Gravity-Compensating Orthosis on Reaching After Stroke: Evaluation of the Therapy Assistant WREX," Arch. Phys. Med. Rehabil., vol. 89 , no. 11, pp. 2121-2128, 2008, doi: 10.1016/j.apmr.2008.04.022.
[11] J. M. Wagner, A. W. Dromerick, S. A. Sahrmann, and C. E. Lang, "Upper extremity muscle activation during recovery of reaching in subjects with post-stroke hemiparesis," Clin. Neurophysiol., vol. 118, no. 1, pp. 164-176, 2007, doi: 10.1016/j.clinph.2006.09.022.

[12] J. P. A. Dewald and R. F. Beer, "Abnormal joint torque patterns in the paretic upper limb of subjects with hemiparesis," Muscle and Nerve, vol. 24, no. 2. pp. 273-283, 2001, doi: 10.1002/1097-4598(200102)24:2<273::AIDMUS130>3.0.CO;2-Z.

[13] M. Owen, C. Ingo, and J. P. A. Dewald, "Upper extremity motor impairments and microstructural changes in bulbospinal pathways in chronic hemiparetic stroke," Front. Neurol., vol. 8, no. JUN, 2017, doi: 10.3389/fneur.2017.00257.

[14] K. B. Wilkins, M. Owen, C. Ingo, C. Carmona, J. P. A. Dewald, and J. Yao, "Neural plasticity in moderate to severe chronic stroke following a device-assisted task-specific arm/hand intervention," Front. Neurol., vol. 8, no. JUN, pp. 1-11, 2017, doi: 10.3389/fneur.2017.00284.

[15] K. B. Wilkins, J. Yao, M. Owen, H. Karbasforoushan, C. Carmona, and J. P. A. Dewald, "Limited capacity for ipsilateral secondary motor areas to support hand function post-stroke," J. Physiol., vol. 598, no. 11, pp. 2153-2167, 2020, doi: 10.1113/JP279377.

[16] S. N. Baker, "The primate reticulospinal tract, hand function and functional recovery," $J$. Physiol., vol. 589, no. 23, pp. 5603-5612, 2011, doi: 10.1113/jphysiol.2011.215160.

[17] A. C. Nirkko et al., "Different ipsilateral representations for distal and proximal movements in the sensorimotor cortex: Activation and deactivation patterns," Neuroimage, vol. 13, no. 5, pp. 825-835, 2001, doi: 10.1006/nimg.2000.0739.

[18] D. M. Miller, C. S. Klein, N. L. Suresh, and W. Z. Rymer, "Asymmetries in vestibular evoked myogenic potentials in chronic stroke survivors with spastic hypertonia: Evidence for a vestibulospinal role," Clin. Neurophysiol., vol. 125 , no. 10 , pp. 2070-2078, 2014, doi: 10.1016/j.clinph.2014.01.035.

[19] V. Smith, D. Maslovat, N. M. Drummond, J. Hajj, A. Leguerrier, and A. N. Carlsen, "Highintensity transcranial magnetic stimulation reveals differential cortical contributions to prepared responses," J. Neurophysiol., vol. 121, 
no. 5, pp. 1809-1821, 2019, doi:

10.1152/jn.00510.2018.

[20] A. G. Davidson, M. H. Schieber, and J. A. Buford, "Bilateral spike-triggered average effects in arm and shoulder muscles from the monkey pontomedullary reticular formation," $J$. Neurosci., vol. 27, no. 30, pp. 8053-8058, 2007, doi: 10.1523/JNEUROSCI.0040-07.2007.

[21] R. K. Powers, D. L. Campbell, and W. Z. Rymer, "Stretch reflex dynamics in spastic elbow flexor muscles," Ann. Neurol., vol. 25, no. 1 , pp. 32-42, 1989, doi: 10.1002/ana.410250106.

[22] G. Hoffmann, M. O. Conrad, D. Qiu, and D. G. Kamper, "Contributions of voluntary activation deficits to hand weakness after stroke," Top. Stroke Rehabil., vol. 23, no. 6, pp. 384-392, 2016, doi: 10.1179/1945511915Y.0000000023.

[23] J. Chae, G. Yang, B. K. Park, and I. Labatia, "Delay in initiation and termination of muscle contraction, motor impairment, and physical disability in upper limb hemiparesis," Muscle and Nerve, vol. 25, no. 4, pp. 568-575, 2002, doi: $10.1002 /$ mus. 10061 .

[24] G. Pfurtscheller, K. Zalaudek, and C. Neuper, "Event-related beta synchronization after wrist, finger and thumb movement," Electroencephalogr. Clin. Neurophysiol. Electromyogr. Mot. Control, vol. 109, no. 2, pp. 154-160, 1998, doi: 10.1016/S0924980X(97)00070-2.

[25] N. J. Seo, W. Z. Rymer, and D. G. Kamper, "Delays in grip initiation and termination in persons with stroke: Effects of arm support and active muscle stretch exercise," $J$.

Neurophysiol., vol. 101, no. 6, pp. 3108-3115, 2009, doi: 10.1152/jn.91108.2008.

[26] S. Balasubramanian, A. Melendez-Calderon, and E. Burdet, "A robust and sensitive metric for quantifying movement smoothness," IEEE Trans. Biomed. Eng., vol. 59, no. 8, pp. 21262136, 2012, doi:

10.1109/TBME.2011.2179545.

[27] F. do Vale Garcia, M. J. da Cunha, C. P. Schuch, G. P. Schifino, G. Balbinot, and A. S. Pagnussat, "Movement smoothness in chronic post-stroke individuals walking in an outdoor environment-A cross-sectional study using IMU sensors," PLoS One, vol. 16, no. 4 April 2021, pp. 1-18, 2021, doi:

10.1371/journal.pone.0250100.

[28] E. E. Fetz, P. D. Cheney, K. Mewes, and S.
Palmer, "Control of forelimb muscle activity by populations of corticomotoneuronal and rubromotoneuronal cells," Prog. Brain Res., vol. 80, no. C, pp. 437-449, 1989, doi: 10.1016/S0079-6123(08)62241-4.

[29] M. Jeannerod, "The formation of finger grip during prehension. A cortically mediated visuomotor pattern," Behav. Brain Res., vol. 19, no. 2, pp. 99-116, 1986, doi: 10.1016/01664328(86)90008-2.

[30] C. E. Lang et al., "Deficits in grasp versus reach during acute hemiparesis," Exp. Brain Res., vol. 166, no. 1, pp. 126-136, 2005, doi: 10.1007/s00221-005-2350-6.

[31] P. Archambault, P. Pigeon, A. G. Feldman, and M. F. Levin, "Recruitment and sequencing of different degrees of freedom during pointing movements involving the trunk in healthy and hemiparetic subjects," Exp. Brain Res., vol. 126, no. 1, pp. 55-67, 1999, doi: $10.1007 / \mathrm{s} 002210050716$.

[32] D. J. Reinkensmeyer, A. McKenna Cole, L. E. Kahn, and D. G. Kamper, "Directional control of reaching is preserved following mild/moderate stroke and stochastically constrained following severe stroke," Exp. Brain Res., vol. 143, no. 4, pp. 525-530, 2002, doi: 10.1007/s00221-002-1055-3.

[33] S. Choudhury et al., "The Relationship Between Enhanced Reticulospinal Outflow and Upper Limb Function in Chronic Stroke Patients," Neurorehabil. Neural Repair, vol. 33, no. 5, pp. 375-383, 2019, doi: $10.1177 / 1545968319836233$.

[34] B. Y. S. A. Edgley, J. A. Eyret, R. N. Lemon, and S. Millert, "Excitation of the Corticospinal Tract By," pp. 301-320, 1990.

[35] J. E. Fox, "Reticulospinal neurones in the rat," Brain Res., vol. 23, no. 1, pp. 35-40, 1970, doi: 10.1016/0006-8993(70)90347-1.

[36] M. R. Senesh, K. Barragan, and D. J. Reinkensmeyer, "Rudimentary Dexterity Corresponds With Reduced Ability to Move in Synergy After Stroke: Evidence of Competition Between Corticoreticulospinal and Corticospinal Tracts?," Neurorehabil. Neural Repair, vol. 34, no. 10, pp. 904-914, 2020, doi: $10.1177 / 1545968320943582$.

[37] J. Bertelli, F. Soldado, and M. F. Ghizoni, "Outcomes of Radial Nerve Grafting In Children After Distal Humerus Fracture," $J$. Hand Surg. Am., vol. 43, no. 12, pp. 1140.e1- 
1140.e6, 2018, doi: 10.1016/j.jhsa.2018.04.018.

[38] D. G. Kamper, H. C. Fischer, E. G. Cruz, and

W. Z. Rymer, "Weakness Is the Primary

Contributor to Finger Impairment in Chronic

Stroke," Arch. Phys. Med. Rehabil., vol. 87, no.

9, pp. 1262-1269, 2006, doi:

10.1016/j.apmr.2006.05.013. 